\title{
Rapid prototyping MEMS with Laminated Resin Printing
}

\author{
Harrison B. Jones ${ }^{\mathrm{a}}$, Ciaran P. Moore ${ }^{\mathrm{b}}$, Andrew D. Best ${ }^{\mathrm{a}}$, Andrea J. Bubendorfer ${ }^{\mathrm{a}}$, and Neil D. \\ Glasson $^{\mathrm{a}}$ \\ ${ }^{a}$ Callaghan Innovation, Lower Hutt, New Zealand \\ ${ }^{\mathrm{b}}$ University of Canterbury, Christchurch, New Zealand
}

\begin{abstract}
Interest in MEMS fabrication has been growing for the last 50 years; however, the number and quality of tools supporting rapid prototyping in this industry are limited. Laminated resin printing (LRP) combines maskless projector-based lithography with 3D printing techniques to facilitate the rapid production of high-resolution polymer structures in three dimensions with no retooling required. By selectively metalising these structures, high value functional MEMS devices can be rapidly produced, tested and iterated. Microcircuits, enclosed mechanical systems and devices based on cantilevers and membranes have all been demonstrated using this technique.
\end{abstract}

Keywords: Laminated Resin Printing, Polymer MEMS, MEMS, Microsystems, Microstructures, Micro 3D printing, Microfluidics, Soft Lithography

\section{INTRODUCTION}

The initial micro-electromechanical systems (MEMS) market was developed in silicon, using techniques based on the existing integrated circuit industry. ${ }^{1,2}$ Silicon MEMS has continued to dominate due to the existing infrastructure, common manufacturing techniques, well characterised material behaviour and desirable electromechanical properties. ${ }^{3}$ Despite this, silicon is not suitable for all applications and suffers from high manufacturing costs, especially for low volume production. ${ }^{3,4}$ Polymer based MEMS, which has been gaining popularity since the early $2000 \mathrm{~s}^{5,6}$ with ink jet nozzles, ${ }^{7}$ microfluidic moulds, ${ }^{8}$ and imprint techniques ${ }^{9}$ offers several advantages over traditional silicon MEMS. Polymer MEMS are more environmentally friendly and cheaper to produce ${ }^{3,4}$ and exhibit many advantageous physical properties including flexibility, chemical resistance and biocompatibility. ${ }^{10}$ However, polymer MEMS are currently lacking in viable paths to manufacturing, ${ }^{11,12}$ while both polymer and silicon MEMS lack rapid prototyping tools. ${ }^{13,14}$ We present Laminated Resin Printing (LRP) as a viable solution to both problems, allowing for complex structures to be produced at low cost with a fast cycle time. With complementary existing microfabrication techniques creates a direct path to fabricate polymer MEMS devices, enabling low volume and custom MEMS production. The technique also allows for rapid prototyping of devices manufactured using traditional techniques. LRP does not require a large manufacturing plant, with the current prototype having a desktop form factor. This can help move MEMS fabrication away from large foundries into a more distributed model. This paper will first define how LRP differs from conventional microfabrication and 3D printing techniques. It will then describe the current prototype system and its performance. We then present a series of example prints produced to show the unique capabilities of the technology.

Further author information: (Send correspondence to H.B.J.)

H.B.J.: E-mail: Harrison.Jones@callaghaninnovation.govt.nz

C.P.M.: E-mail: Ciaran.Moore@canterbury.ac.nz

A.J.B.: E-mail: Andrea.Bubendorfer@callaghaninnovation.govt.nz

A.D.B.: E-mail: Andrew.Best@callaghaninnovation.govt.nz

N.D.G.: E-mail: Neil.Glasson@callaghaninnovation.govt.nz 


\section{BACKGROUND}

Laminated Resin Printing is a new MEMS prototyping process that uses stacks of patterned dry resin in aligned layers to fabricate 3D microstructures. Each layer of the stack is patterned using photolithography - which is a pattern transfer technique using photoresist, a material in which solubility changes after being exposed to optical radiation. When a negative photoresist is exposed to ultraviolet (UV) light and then heated, it becomes cross-linked and therefore much less soluble. Because of their insolubility, cross-linked negative photoresists are permanent. The patternability of permanent thick structures, along with their low Young's Modulus (typical range of $2-4 \mathrm{GPa},{ }^{15}$ compared to 130 - $180 \mathrm{GPa}$ for Silicon ${ }^{16}$ ), chemical resistance and ease of use make negative photoresists an ideal structural material for polymer MEMS.

LRP forms microstructures from dry film negative photoresist in a rapid automated process, significantly reducing workload over traditional microfabrication techniques. The resist layers are packaged between cover sheets to protect from contamination, and are here used for transport during processing. Each layer is individually patterned as a section of film, using a maskless UV source, such as a laser or projector, as shown in 1. After patterning, the first layer is laminated to a substrate that acts as a print bed; and any subsequent layers are laminated to the previous layer. This builds up a block of resin containing the patterned structure. Building in the uncrosslinked material provides inherent support for printing membranes and overhangs. After all layers are sequentially patterned and laminated to the bulk, the structure is cross-linked and developed. Upon development the structure is complete. If required additional post processing steps such as metallisation, etching or other microfabrication techniques, can take place.

LRP solves several issues with traditional polymer MEMS, including improving process uniformity and film thickness through the use of dry films, and improving adhesion by using lamination. As negative resist based structures are permanent the structures produced with LRP are not limited to being only an intermediate manufacturing step. LRP's main advantage is the low cycle time and its ability to fabricate complex 3D structures.

\section{DESCRIPTION OF CURRENT SYSTEM}

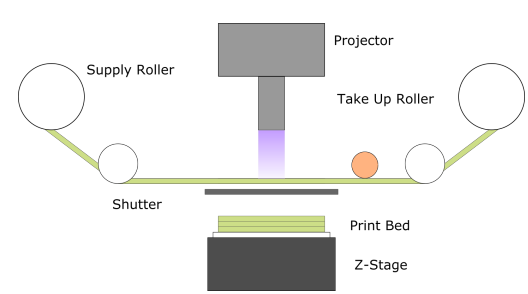

(a)

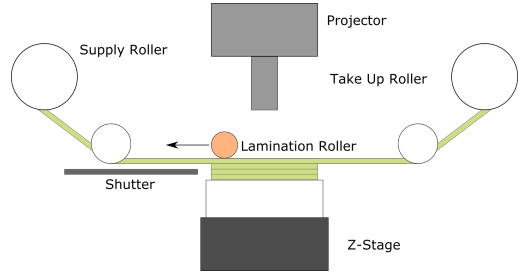

(b)

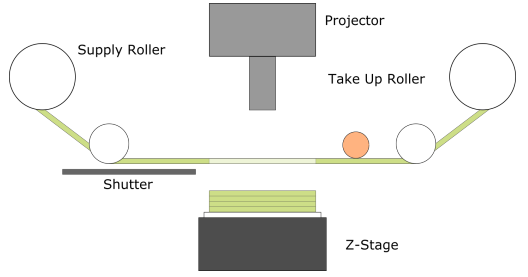

(c)

Figure 1: The print sequence for the current LRP system. (a) The projector exposes the active layer with a shutter protecting the preceding layers which are already laminated to the $\mathrm{Z}$ stage. (b) The shutter is removed and the $\mathrm{Z}$ stage and preceding layers are moved up to the active layer. A heated lamination roller then laminates the active layer to the preceding layers. (c) The Z stage retracts, freeing the last layer from the cover sheet. The roll advances and the shutter is replaced to repeat the process.

The current system utilises a DLP based Visitech LRS projector ${ }^{17}$ to enable rapid maskless patterning of the photoresist. With a maskless system, structures are defined in software, removing the need for retooling between printing different designs, reducing cost and cycle time. It also removes the need for a mechanism for changing masks between layers and improves alignment. The system can be configured to either give $5 \mu \mathrm{m}$ square pixels or $10 \mathrm{\mu m}$ pixels, depending on the optics used in front of the projector. As the current projector has a resolution of $1920 \times 1080$, working area is dependent on pixel size. In the $10 \mu \mathrm{m}$ configuration the working area is $19.2 \mathrm{~mm}$ $\mathrm{x} 10.8 \mathrm{~mm}$.

The printing process can be seen in Figure 1. Photoresist film is fed from a roll in a UV excluding enclosure to the patterning area. The resist is then patterned above the substrate with a shutter protecting any preceding layers. The layer being patterned is referred to as the active layer. After patterning, the resist is laminated to 
the preceding layers or substrate. A high resolution $\mathrm{Z}$ stage is used to hold the bulk and pull the resist from the tape after lamination. The remaining, waste film on the transport layer is then fed to a take-up roll and fresh resist is brought to the pattern area to repeat the sequence.

\subsection{Current System Performance}

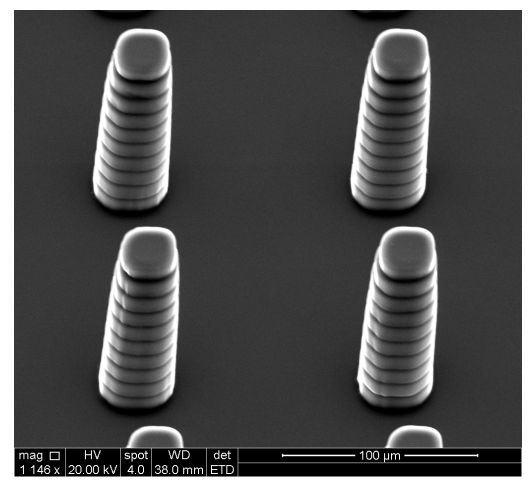

(a)

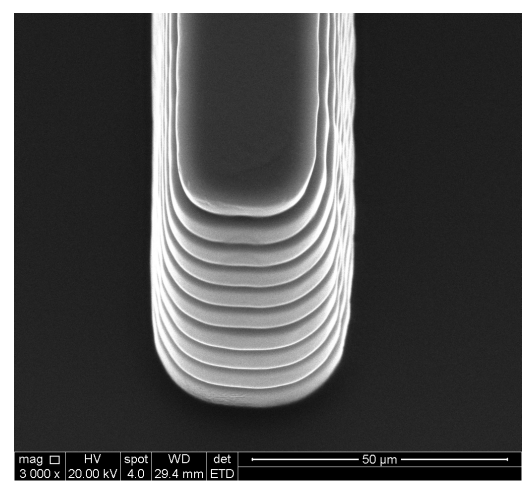

(c)

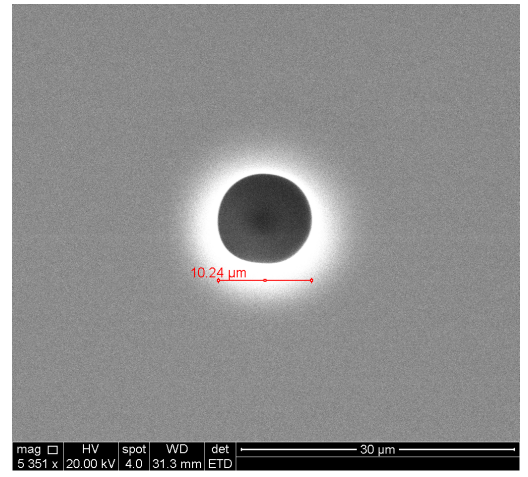

(b)

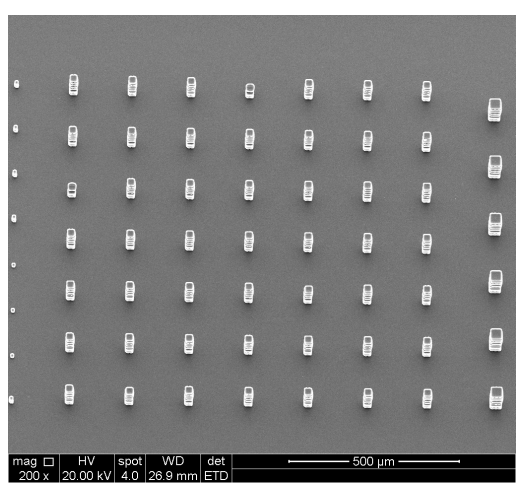

(d)

Figure 2: (a) Four $20 \mu \mathrm{m}$ wide and $100 \mu \mathrm{m}$ micron tall pillars manufactured by laminating then exposing. (b) A $10 \mu \mathrm{m}$ pore in a $10 \mu \mathrm{m}$ thick membrane. (c) A $30 \mu \mathrm{m}$ wide $100 \mu \mathrm{m}$ tall wall also manufactured with laminate then expose, showing tapered sidewalls. (d) An array of $20 \mu \mathrm{m}$ wide $100 \mu \mathrm{m}$ tall pillars using the expose then laminate method with vertical sidewalls.

With the current $1920 \times 1080$ pixel system, LRP is capable of achieving $10 \mu \mathrm{m}$ true resolution. In this system, resolution is defined as the size of a pixel projected onto a resist layer. The minimum positive feature size, defined as the diameter of the smallest possible printed pillar, is $20 \mu \mathrm{m}$, as shown in Figure 2 (a). The minimum negative feature size is defined as the smallest pore producible, which as shown in Figure 2 (b) has a diameter of $10 \mu \mathrm{m}$. Aspect ratio is an important concern in microfabrication, and especially with lithography. The current system is capable of operating in two modes for multilayer structures; laminate and then expose, and expose and then laminate. When exposing and then laminating a shutter can be placed between the active layer and the preceding layers. This stops light exposing earlier layers and enables extreme overhangs. The advantage of laminating first is that the active layer is locked in position before exposure, improving alignment. Layer mis-alignment is dependent on the repeatability of the stage and is currently $<2 \mu \mathrm{m}$. This also improves layer adhesion by improving cross linking between layers, especially at the edge of structures that may receive less light, which can cause undercutting. The disadvantage is that overhangs of any angle are impossible as the exposure of the active layer would also expose earlier layers. Scattered light also decreases the sidewall angle of the overall structure in this mode. Figure 2 (c) shows a wall produced in this mode with a sidewall angle of $86^{\circ}$. Figure $2(\mathrm{~d})$ shows the same pattern as (a) but using expose then laminate to achieve vertical sidewalls. 
While tapered sidewalls may be detrimental to some applications, they are ideal for moulding and embossing applications.

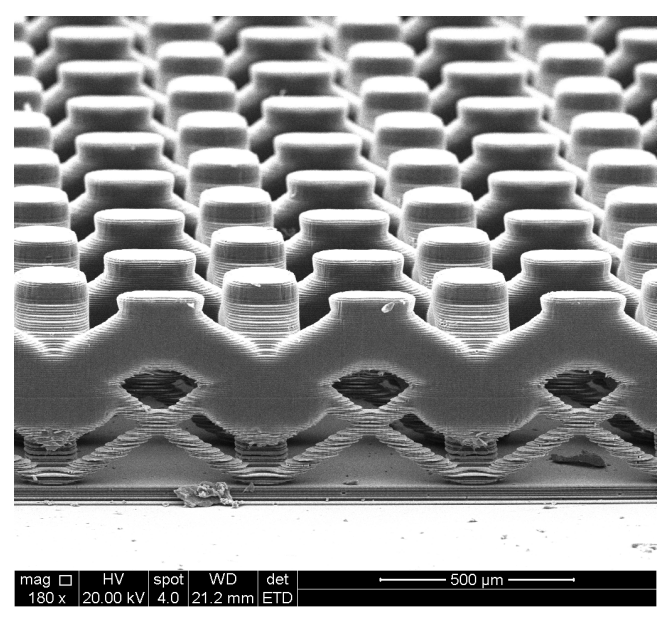

Figure 3: A lattice where the lower layers were manufactured using the expose then laminate method while the top layers used laminate then expose. Over penetration to lower layers is visible.

Figure 3 shows a lattice structure where the bottom half was produced using the laminate then expose method while the top half was produced by exposing and then laminating. Over-penetration can be seen through the top layers, however the top of these layers exhibit less undercutting than the layers at the bottom. This exhibits the advantages and disadvantages of each print mode.

As each layer can be patterned individually and then stacked, LRP is capable of producing incredibly high aspect ratio structures. The main limitation with the current system is the resolution of the projector, with the structure in Figure 2 (a) having an aspect ratio of 5. The maximum print height of a structure is limited only by the travel of the linear Z stage, currently $50 \mathrm{~mm}$, and its alignment. At this time features as large as $2.2 \mathrm{~mm}$ have been demonstrated. As the system is automated, print time is very low and once a print is started requires no input from the user. Less than five minutes are required to load design files and set up the system. Each individual layer takes 45 seconds to expose and laminate, so a $2 \mathrm{~mm}$ tall structure takes less than three hours to print. Development time is dependent on the structure's shape but can be as low as 10 minutes up to around an hour.

\section{EXAMPLE PRINTS}

\subsection{Cantilevers and Overhangs}

As a particular advantage of the LRP process, overhangs of any angle can be easily produced ${ }^{18}$ as structures are printed within their own support material. This can be seen in Figure 4. These overhangs can be used to print flexible active structures such as cantilevers used as the basis for many simple sensing devices, as in (b) through to entire microsystems suspended from a membrane. The membrane in Figure 4 (a) is one layer thick, in this case $10 \mu \mathrm{m}$, and $500 \mu \mathrm{m} \times 300 \mu \mathrm{m}$ in the $\mathrm{x}, \mathrm{y}$ dimensions. An extension of this is to produce totally free standing structures, either like the microfluidic chips described in Section 4.3, or as free standing mechanical components as in Figure 4 (c). These gears are not attached to the main structure in any way and are only constrained by their axles and the mounting brackets, allowing them to rotate freely. This could be combined with a microcircuit, as discussed in Section 4.5, to create an electrostatic motor or an energy harvesting device. Cantilevers such as those in (b) could also be functionalised and used for a number of sensing applications.

\subsection{Optics}

The photoresist used in the current system has an added dye to increase photospeed, thus making it unsuitable for direct production of optics. LRP can however, be used to manufacture moulds which can be replicated using soft 


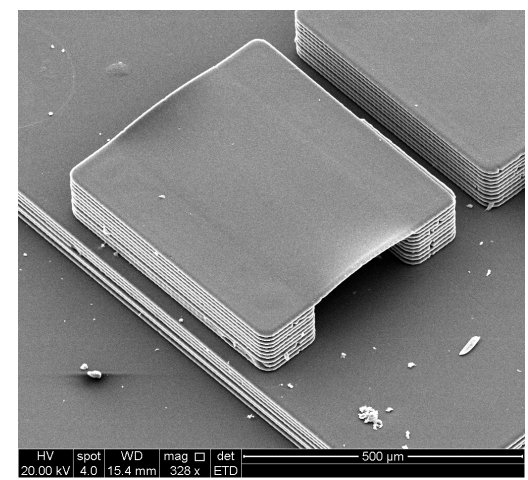

(a)

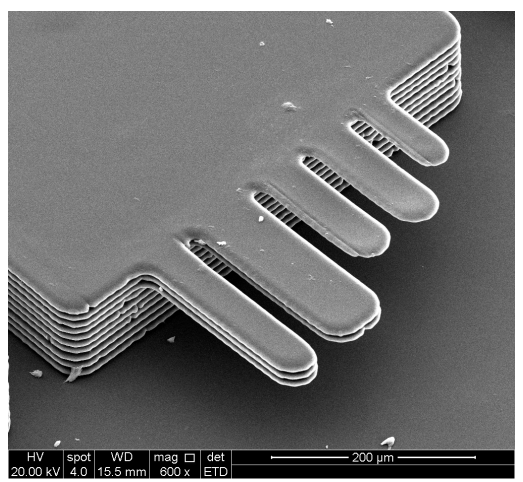

(b)

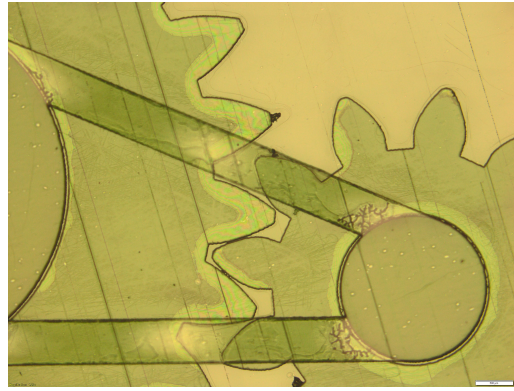

(c)

Figure 4: (a) A $500 \mu \mathrm{m}$ x $300 \mu \mathrm{m}$ membrane suspended $100 \mu \mathrm{m}$ above the substrate (b) A series of cantilevers of varying lengths and thicknesses. The longest are $200 \mu \mathrm{m}$ (c) Free standing gears which can be manually rotated printed as a single part.

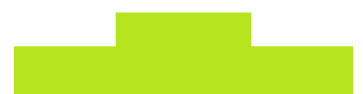

(a)

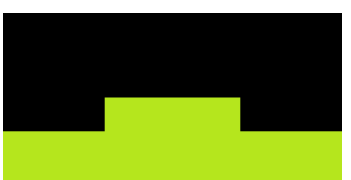

(b)

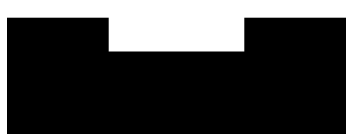

(c)

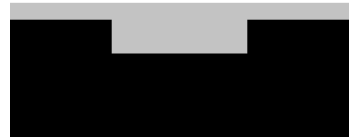

(d)

Figure 5: A waveguide is produced by following soft lithography processing steps. (a) A mould is produced using the LRP prototype. (b) PDMS material A is poured onto the mould and cured. (c) The PDMS casting A is peeled off. (d) PDMS material B is poured onto the PDMS casting and cured. Both PDMS materials A and $\mathrm{B}$ are bond together forming a unit.

lithography with polydimethylsiloxane (PDMS). PDMS is commonly used in soft lithography for prototyping of optical elements due it's high optical transparency. Figure 5 shows the process for creating PDMS structures using an LRP mould. For replicating simple single-material structures, only steps one to three are required. Figure 6 (a) is an example of such a structure, demonstrating the ability to produce transparent optical structures. Figure 6 (b) shows a laser passing through a more complex structure of size varying waveguides produced using the full method in 5. The first PDMS material (poured onto the LRP mould) contained carbon nanotubes to block light, whereas the second PDMS material poured onto the casting contained copper and gold nanoparticles to increase internal light reflection. A limitation of the current system's ability to produce optical elements is the stepped nature due to the layer by layer construction. In the future greyscale lithography techniques could be used to reduce stepping between layers and create sub layer features. Combining this with an optically transparent photoresist would greatly improve the system's capability for manufacturing optics.

\subsection{Microfluidics}

The ability to print complex internal structures lends itself particularly well to microfluidics. Typically when using lithography to produce microfluidics, a negative photoresist is spin coated onto a glass or silicon substrate. This, while a mature technique, has many of the same issues associated with typical microfabrication. It is expensive and, unless specialist equipment is used, it is a time consuming manual process. Additionally when using glass or silicon substrates, reflections can cause loss of resolution. The mass production of these chips is usually achieved with PDMS moulding using standard soft lithographic techniques, another manual and difficult to automate process. ${ }^{19}$ With LRP, complex multilayer microfluidic systems can be rapidly produced. A glass substrate and lid are not needed as the process can fully encapsulate structures.

The microfluidics area is already seeing increasing utilisation of $3 \mathrm{D}$ printing technologies to facilitate rapid prototyping. ${ }^{13,20}$ The most common 3D printing technology in this space is Stereolithography (SLA), which unlike Fused Deposition Modelling (FDM), can easily produce watertight and higher resolution structures. ${ }^{21}$ 

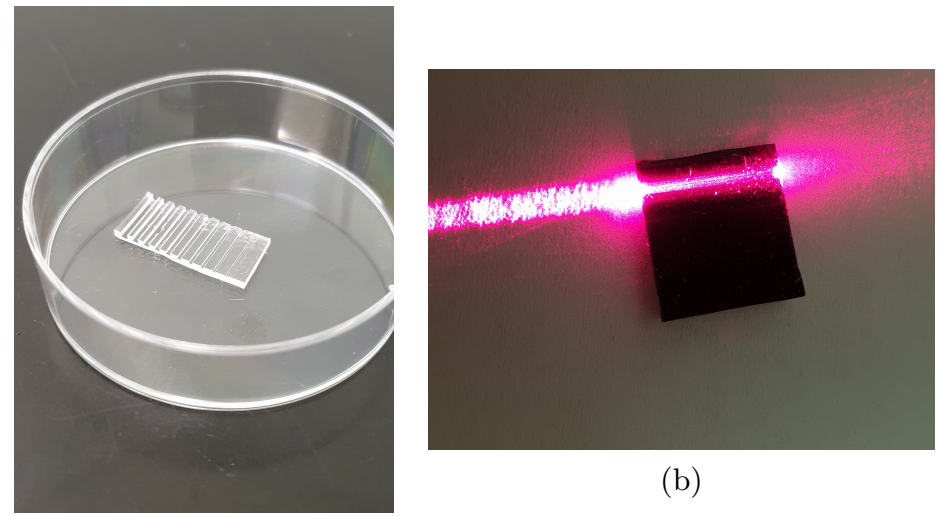

(b)

(a)

Figure 6: (a) Clear PDMS casting of an LRP mould produced by soft lithography. (b) A laser passes through one waveguide in an array produced using the process in Figure 5

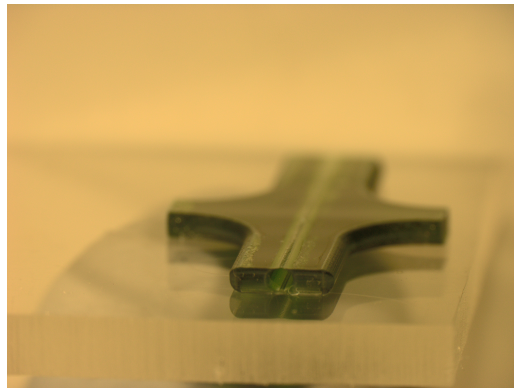

(a)

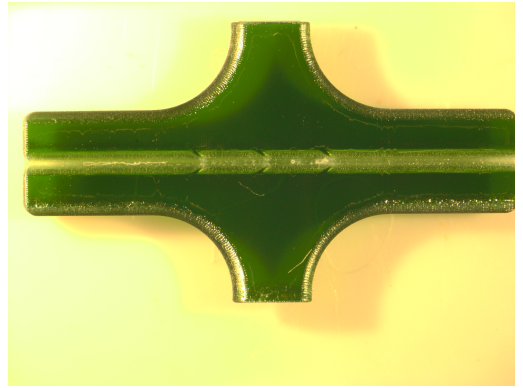

(b)

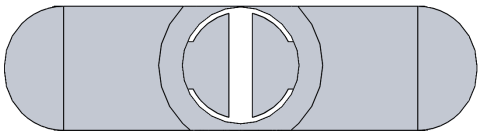

(c)

Figure 7: (a) and (b) Images of a microfluidic chip based on the valves in a human vein produced with LRP out of $5 \mathrm{\mu m}$ layers. Of note is the circular channel and the custom connection ports for $4 \mathrm{~mm}$ piping. (c) A cross section of the chip showing the shape of the valve and the large overhang area.

While these printers are an established technology, they have inherent limitations that have so far prevented them from taking the majority market share away from standard lithographic processes, ${ }^{22}$ despite difficulty in using such processes to create 3D microfluidic systems. SLA printers typically have a resolution of $25 \mu \mathrm{m}$, however actual minimum feature size is usually larger. ${ }^{21}$ They also have difficulty handling steep overhangs and are incapable of producing free standing internal structures. LRP does not share these issues and allows for structures such as those in Figure 7, which is a microfluidic channel with several internal valves, modelled after the valves found within a human vein. Figure 7 (c) shows a cross section of the channel and valve where the negative overhang can be clearly seen. Also of note is that the channel is circular to better model the human vein, this would be difficult to achieve using standard lithographic processes. The ability to create free standing internal structures can also be utilised in microfluidics. An example of this is the micropiston in Figure 8. The piston itself is unconnected from the framing with one $10 \mu \mathrm{m}$ layer clearance above and below the piston and 20 $\mu \mathrm{m}$ clearance either side. This is necessary during the manufacturing process to ensure the piston is free of the cylinder. With this clearance the seal is good enough to use the piston either with water of even pneumatically. Sealing could be improved by using a higher resolution exposure source and by decreasing the layer size. 5 $\mu \mathrm{m}$ layers have since been demonstrated in structures such as in Figure 7. The main limitation to this kind of internal structures is development. It takes a significant amount of time for the developer to fully remove all uncross linked material in large chambers at the end of narrow channels such as in this case. This was improved by adding development holes to allow more access points for the developer. These can be seen as the circles within the cylinder cavity. These were then sealed by laminating another layer of resist to the bottom of the structure manually and cross linking. 


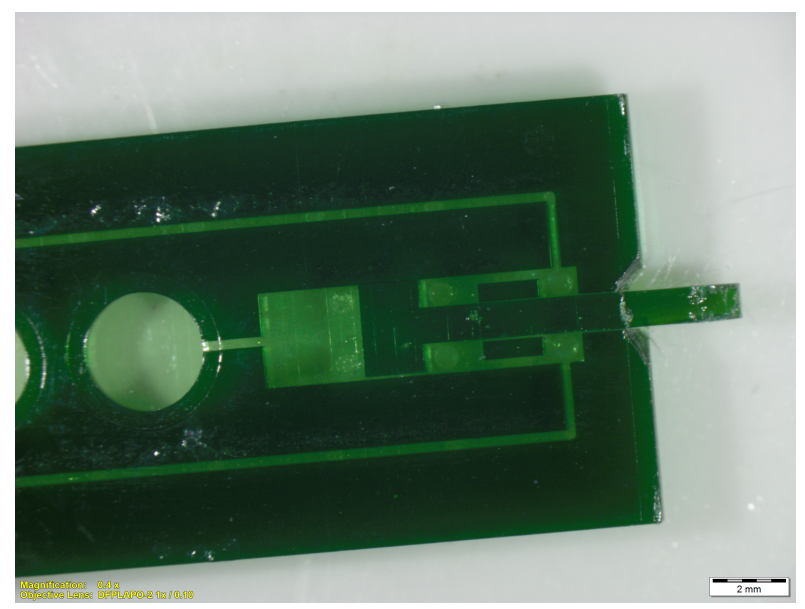

Figure 8: A microfluidic/pneumatic piston with an actuating internal structure. This was printed as a single part utilising LRP's inherent removable support material from uncrosslinked photoresist.

\subsection{Shadow Masks}

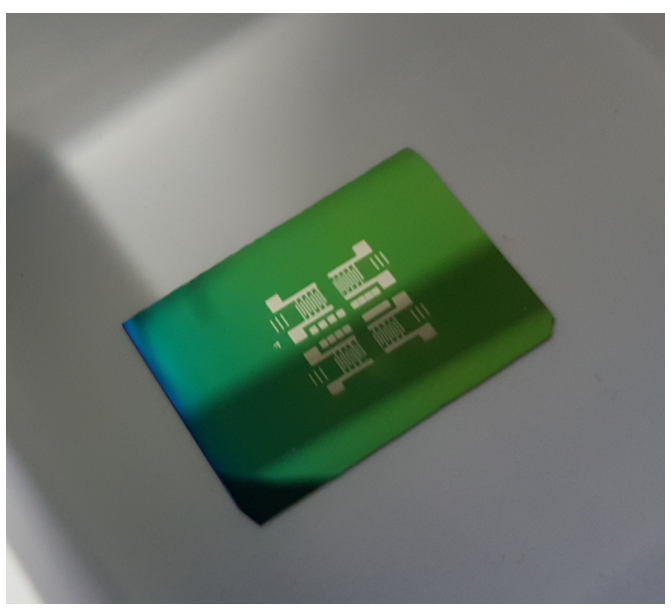

Figure 9: Interdigitated electrodes created by ion-beam sputtering using a polymer shadow mask which was manufactured using the LRP prototype. Credit to Holger Fiedler, Jérôme Leveneur, Materials Team, GNS Science, New Zealand

Shadow masks are used for transferring a pattern to a substrate using sputtering or similar techniques. Standard metal shadow masks can introduce contaminants into the sample. LRP's ability to pattern free standing membranes makes it ideal for producing polymer shadow masks. When used with antimony free photoresists these can be completely metal free. Figure 9 shows an example of the types of designs possible using LRP shadow masks.

\subsection{Microcircuits}

Additional functionality for further MEMS applications can be added with complementary techniques. Parts can be metalised by first printing a structure with LRP as normal. The structure is then coated on one or both sides with a metal film by e-beam evaporation or sputtering. This metal film is separated into multiple nets using the shape of the structure, as seen in Figure 10 (a) creating an electric circuit. An alternative method, shown in Figure 10 (b), is to metalise as in the first method but to then remove the top layer of metal. This is 


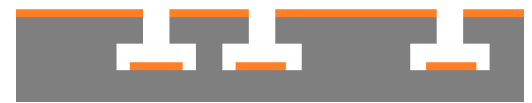

(a)

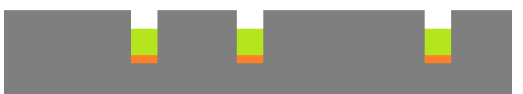

(b)

Figure 10: (a) Metal coating with multiple isolated nets produced by evaporating metal onto a resist structure. (b) An alternate method where the surface metal has been etched away to improve isolation between nets.

accomplished by filling the channels/traces with a photoresist and then etching. As the resist is thicker over the traces, the top metal layer is removed first, leaving only the metal within the channels. These methods allow for the creation of both capacitive and resistive sensors, and electrostatic actuators. The low number of steps required, and high process automation lowers both cost and cycle time for prototyping such devices and provides a pathway for rapid development of active MEMS devices.

\section{CONCLUSION}

This research has shown the potential for LRP to be used in the MEMS industry, providing a unique set of capabilities that may augment existing processes and aid in rapid prototyping; or function as a manufacturing process in its own right. LRP provides a low-cost, fast turn-around opportunity to further development of new MEMS devices, especially polymer MEMS, with properties advantageous for many applications including those requiring elasticity, chemical resistance or low cost. A number of devices were demonstrated including microfluidics, optics, cantilevers and membranes which themselves form the basis of the common MEMS springs used for many sensor components, shadow masks and microcircuits. Existing off-the-shelf solutions provide a direct path for increased resolution and print area, while addition of complementary techniques such as greyscaling and metalisation provide further opportunities for MEMS development.

\section{ACKNOWLEDGMENTS}

The authors would like to thank Callaghan Innovation for financial support to fund this project, and acknowledge the contributions from Dr Andreas Zeller for assistance with optical devices, the Callaghan Innovation Christchurch engineering team for excellence in engineering developing the prototype and GNS as mentioned for the use of the shadow mask image.

\section{REFERENCES}

[1] LaBianca, N. C. and Gelorme, J. D., "High-aspect-ratio resist for thick-film applications," in [Advances in Resist Technology and Processing XII], Allen, R. D., ed., 2438, 846 - 852, International Society for Optics and Photonics, SPIE (1995).

[2] Shaw, J. M., Gelorme, J. D., LaBianca, N. C., Conley, W. E., and Holmes, S. J., "Negative photoresists for optical lithography," IBM Journal of Research and Development 41, 81-94 (Jan 1997).

[3] Li, Y., "Challenges and issues of using polymers as structural materials in MEMS: A review," Journal of Microelectromechanical Systems 27, 581-598 (Aug 2018).

[4] Hussain, F., "MEMS evolution: from Silicon to Polymers." TTP, 21 August 2017 https://www.ttp.com/ $\mathrm{blog} / \mathrm{mems}$-evolution-from-silicon-to-polymers. (Accessed: 8 January 2020).

[5] Fitzgerald, A. M., "The Internet of Disposable Things Will Be Made of Paper and Plastic Sensors." Overleaf, 26 February 2015 https://spectrum.ieee.org/semiconductors/materials/ the-internet-of-disposable-things-will-be-made-of-paper-and-plastic-sensors. (Accessed: 8 January 2020).

[6] Bertsch, A. and Renaud, P., "Special issue: 15 years of su8 as MEMS material," Micromachines 6, 790-792 (Jun 2015).

[7] Yi, M., Feng, J., Yin, Z., and Zou, H., "Fabricating method of su-8 photoresist conical nozzle for inkjet printhead," Materials and Manufacturing Processes 33(8), 898-904 (2018). 
[8] Garcia, C., Lang, W., Schomacker, A., Klarholz, I., and Harms, C., "Fabrication of microfluidic devices using su-8 for detection and analysis of viruses," in [SENSOR+TEST Conferences], Proc. AMA 1, 193-197 (2009).

[9] Shen-Qi Xie, Bing-Rui Lu, Jing Wan, Rong Yang, Yifang Chen, Xin-Ping Qu, and Ran Liu, "Development of novel su-8 based nanoimprint lithography," in [2008 9th International Conference on Solid-State and Integrated-Circuit Technology], 2476-2479 (Oct 2008).

[10] Kotzar, G., Freas, M., Abel, P., Fleischman, A., Roy, S., Zorman, C., Moran, J. M., and Melzak, J., "Evaluation of MEMS materials of construction for implantable medical devices," Biomaterials 23(13), 2737 - 2750 (2002).

[11] Kim, B. J. and Meng, E., "Review of polymer MEMS micromachining," Journal of Micromechanics and Microengineering 26, 013001 (nov 2015).

[12] Liu, C., "Recent developments in polymer MEMS," Advanced Materials 19(22), 3783-3790 (2007).

[13] Bhushan, B. and Caspers, M., "An overview of additive manufacturing (3d printing) for microfabrication," Microsystem Technologies 23, 1117-1124 (3 2017).

[14] Madou, M. J., [Manufacturing Techniques for Microfabrication and Nanotechnology], CRC Press, Boca Raton (2011 (First edition)).

[15] Lorenz, H., Despont, M., Fahrni, N., LaBianca, N., Renaud, P., and Vettiger, P., "SU-8: a low-cost negative resist for MEMS," Journal of Micromechanics and Microengineering 7, 121-124 (sep 1997).

[16] Hopcroft, M. A., Nix, W. D., and Kenny, T. W., "What is the young's modulus of silicon?," Journal of Microelectromechanical Systems 19, 229-238 (April 2010).

[17] "LUXBEAM Rapid System LRS-WQ." Visitech https://visitech.no/lrs-wq/. (Accessed: 8 January 2020).

[18] Mata, A., Fleischman, A. J., and Roy, S., "Fabrication of multi-layer SU-8 microstructures," Journal of Micromechanics and Microengineering 16, 276-284 (jan 2006).

[19] Pimenta, S., Ribeiro, J. F., Goncalves, S. B., Maciel, M. J., Dias, R. A., Gaspar, J., Wolffenbuttel, R. F., and Correia, J. H., "Su-8 based waveguide for optrodes," Proceedings 2(13) (2018).

[20] Bhattacharjee, N., Urrios, A., Kang, S., and Folch, A., "The upcoming 3d-printing revolution in microfluidics," Lab Chip 16, 1720-1742 (2016).

[21] Macdonald, N. P., Cabot, J. M., Smejkal, P., Guijt, R. M., Paull, B., and Breadmore, M. C., "Comparing microfluidic performance of three-dimensional (3d) printing platforms," Analytical Chemistry 89(7), 38583866 (2017). PMID: 28281349.

[22] Waheed, S., Cabot, J. M., Macdonald, N. P., Lewis, T., Guijt, R. M., Paull, B., and Breadmore, M. C., "3d printed microfluidic devices: enablers and barriers," Lab Chip 16, 1993-2013 (2016). 